\section{Correlation between mandibular trabeculae bone density on panoramic radiograph and body mass index of men aged 5-35 years old}

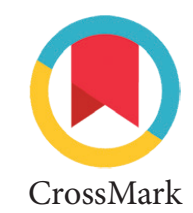

\author{
Binar Barlian, * Azhari, Farina Pramanik
}

\title{
Abstract
}

Objective: Finding the correlation between mandibular trabeculae bone density on panoramic radiograph and body mass index of men aged 5 -35 years old.

Material and Methods: The population of this study was male patients aged 5-35 years old who performed panoramic radiography at Radiology Installation of Faculty of Dentistry at Dental Hospital Padjadjaran University (UNPAD) with a total of 62 samples. ImageJ software was used to calculate the bone density and BMI values were calculated using the BMI formula.

Results: The score of body mass index increased with age meanwhile the score of mandibular trabeculae bone density decreased.

Conclusion: The r-value of this study was -0.341 . There was no correlation between mandibular trabeculae bone density on panoramic radiographs and body mass index of men aged 5-35 years old.
Department of Radiology, Faculty of Dentistry, Padjadjaran University, Bandung, Indonesia
*Corresponding to: Binar Barlian, Department of Radiology, Faculty of Dentistry, Padjadjaran University, Bandung, Indonesia binarbarlian@gmail.com

Received: 18 July 2018 Revised: 10 October 2018 Accepted: 7 November 2018 Available Online 1 December 2018

Keywords: Mandibular trabeculae bone density, Panoramic radiograph, Body mass index

Cite this Article: Barlian B, Azhari, Pramanik F. 2018. Correlation between mandibular trabeculae bone density on panoramic radiograph and body mass index of men aged 5-35 years old. Journal of Dentomaxillofacial Science 3(3): 166-168. D0I: 10.15562/jdmfs.v3i3.777

\section{Introduction}

Body Mass Index (BMI) is an index of height and weight relationships commonly used to classify overweight and underweight conditions. BMI is calculated by the weight $(\mathrm{kg})$ divided by squared height in meters $\left(\mathrm{m}^{2}\right) .{ }^{1}$

Bone mass is the weight of bone substance per unit of external volume. Approximately, the content of bone is $50-70 \%$ mineral that is largely hydroxyapatite, therefore, bone mass is also called bone density or Bone Mineral Density (BMD). One of the most affected bone mineral density from body mass index is the vertebral bone mineral density. Research showed a significant correlation between height, weight and BMI with the development and posture of spinal bone. ${ }^{2}$

The correlation between bone mass density of the mandible and the skeletal bone had been extensively investigated and most of them show there were correlations between the density of both bones. ${ }^{3}$ The bone mass density of the mandible could be observed from the cortical or trabecular regions. The area chosen for this study was the trabeculae because of its rapid response to metabolic changes.

The relationship between mandibular bone density and BMI is controversial, as some studies suggested that the relationship between both are minimal or nonexistent, whereas some studies suggested that both are interconnected.

The purpose of this study was to determine the correlation between mandibular trabeculae bone density and BMI.

\section{Material and Methods}

The researchers will focus on the mandibular trabeculae bone density and body mass index of men aged 5-35 years old. Men were chosen based on the few studies involving mandibular bone density in men. The size of the mandible as a whole increases during growth during puberty ${ }^{4}$ and continues to increase until peak growth period by the age of $35 .^{5}$ Body mass index will continue to increase throughout childhood and adolescence. ${ }^{6}$

Researchers used panoramic radiology to observe the condition of mandibular trabeculae bone density. Panoramic radiographs have been widely used to obtain a comprehensive picture of the structure of the maxillofacial complex. ${ }^{7}$ Some qualitative and quantitative indices, such as the mandibular cortical index, mandibular cortical thickness or mandibular panoramic index, use panoramic radiograph to assess the bone quality. ${ }^{8}$

This study uses primary data in the form of panoramic radiographs. The population and sample of this study is male patients aged 5-35 years old who have done Dental Hospital Padjajaran University Panoramic Radiography at Dental Radiology Installation between January and February 2018.

The inclusion criteria were mongoloid Indonesian, good quality panoramic radiographs and good quality mental foramen area. The exclusion criteria were panoramic radiographs with lesions in the region of interest (mental region), panoramic radiographs of mental foramen superimposed with hyoid bone image and panoramic radiographs 
showing fractures in the area of the mental foramen.

The trabecular density of the mandible was measured using ImageJ software, by measuring the trabecular patterns of panoramic radiographs. The procedures were selecting the Region of Interest (ROI) by using a rectangle tool with a size of $5 \times 5 \mathrm{~mm}$ in the central area of foramen mental, cropping the selected area figure 1, blurring the image using Gaussian blur with 2.00 sigma, subtracting the original image with the blurred image, adding value of 128 then multiply by 1 , converting the image into binary image, eroding

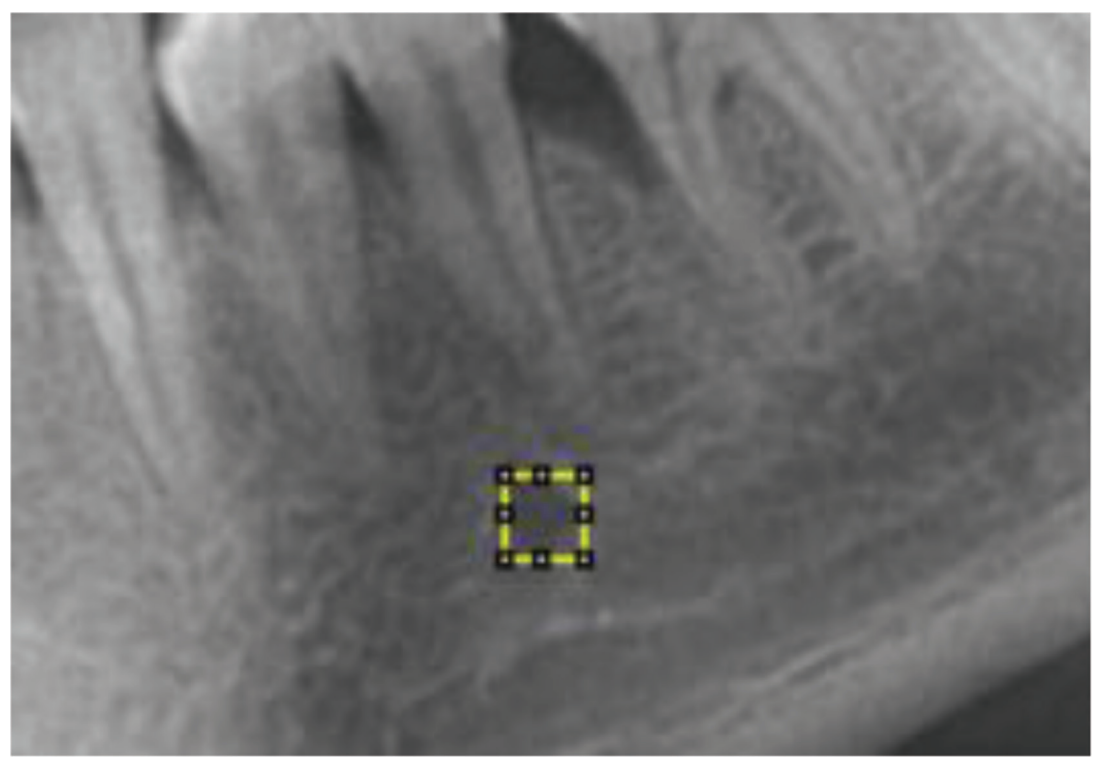

Figure 1 Selecting ROI
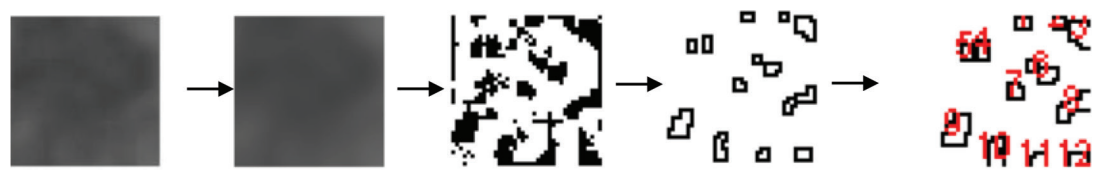

Figure 2 Converting ROI image into outline

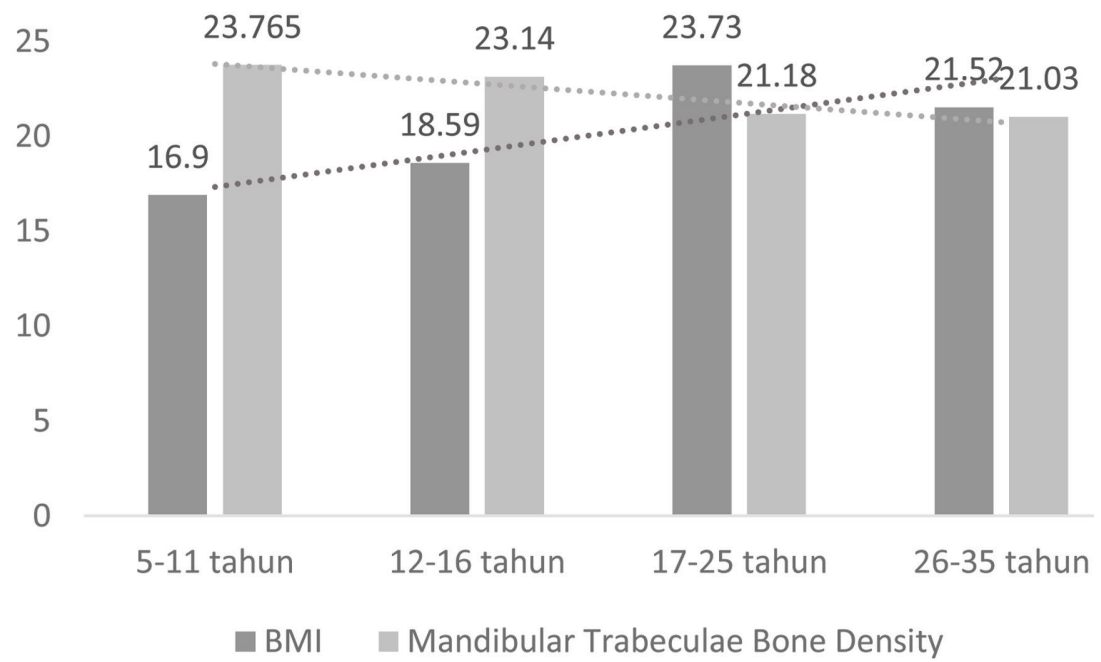

Figure 3 Mean score of mandibular trabeculae bone density and body mass index per age group and dilating the image sequentially by two times, converting the binary image into outline figure 2 , and analyzing the image with analyze particle. The percentage (\%) of the image was the particle of trabecula.

The body mass index was calculated using a stature meter to measure the height and a weight scale to measure the subject's weight. The body mass index was measured by dividing the body weight in kilograms by the height squared in meters $\left(\mathrm{kg} / \mathrm{m}^{2}\right)$.

\section{Results}

There were 62 samples in this study. The samples are divided into age groups according to Indonesia Minister of Health in 2009. The age groups are ages 5-11, ages 12-16, ages 17-25, and ages 26-35. Based on table 1 , the number of samples in each age group can be seen. All age groups had an average of lower trabecular density in the right side compared to the left side. This was caused by the habit of chewing on one side resulting in different density of the mandibular trabecula on both sides. The dominant side of the jaw will have a higher density than the non-dominant side. The average BMI score of all age groups was $20.18 \mathrm{~kg} / \mathrm{m}^{2}$ and the mean score of the mandibular trabecula density of all age groups was $22.23 \%$.

Based on figure 3, we can see the mean of mandibular trabeculae bone density and body mass index for each age group. The BMI scores of the 5-11 age group, 12-16 age group, and the 17-25 age group increased with age. The BMI scores of the 26-35 age group decreased. The mean of mandibular trabeculae bone density decreased from the 5-11 age group to the 26-35 age group.

Based on table 2, we can see the correlation value of BMI and mandibular trabeculae bone density calculated using pearson correlation analysis. The correlation value ( $\mathrm{r}$ - value) was -0.341 . The $\mathrm{p}$-value value of this research was 0.003 .

\section{Discussion}

The results of this study showed mandibular trabeculae bone density decreased with increasing age. This was caused by several factors, such as reduced activity, reduced function of the mandible and reduced steroid hormone. Some other factors such as lifestyle, smoking, and alcohol consumption may also affect the decrease in bone density. ${ }^{9}$

BMI scores increased in the 5-11 age group until the 12-16 age group. This result matched Malina's study in 1999 and Stang and Story's research in 2005. Children gained weight by $2-3 \mathrm{~kg}$ per year when they were around 6-10 years. ${ }^{10}$ Teenagers gained weight by 7-30 kg during puberty. ${ }^{11}$ There 
Table 1 Mean score of mandibular trabeculae bone density and body mass index per age group

\begin{tabular}{lccccc}
\hline & & \multicolumn{4}{c}{ Mean } \\
\cline { 3 - 5 } & & \multicolumn{4}{c}{$\begin{array}{c}\text { Mandibular trabeculae bone } \\
\text { density (\%) }\end{array}$} \\
\cline { 3 - 4 } Age & Sample & BMI $\left(\mathbf{k g} / \mathbf{m}^{2}\right)$ & Right Side & Left Side & Mean \\
\hline $5-11$ & 17 & 16.9 & $23.08 \%$ & $24.45 \%$ & $23.76 \%$ \\
$12-16$ & 9 & 18.59 & $23.06 \%$ & $23.22 \%$ & $23.14 \%$ \\
$17-25$ & 17 & 23.73 & $20.32 \%$ & $22.04 \%$ & $21.18 \%$ \\
$26-35$ & 19 & 21.52 & $20.22 \%$ & $21.85 \%$ & $21.03 \%$ \\
Mean & & 20.18 & & & $22.23 \%$ \\
\hline
\end{tabular}

Table 2 Correlation value of body mass index and mandibular trabeculae bone density

\begin{tabular}{lcc}
\hline Variable & $\mathbf{r}$ & p-value \\
\hline BMI and mandibular trabeculae bone density & -.341 & 0.003 \\
\hline
\end{tabular}

was a significant increase in the 17-25 age group. There were samples with BMI score above 35 in the 17-25 age group. Ages 17-25 years old were very susceptible to weight gain. This was due to several factors such as lifestyle, stress, physical activity and socioeconomic conditions. Decrease in BMI scores was seen at the age of 26-35 years. This is due to overweight samples with high BMI score in the age group of 17-25 years, thus causing the average score of BMI in the 17-25 age group very high.

The results of this study matched the results of Iwaniec and Turner's research in 2014, which stated that people with high BMI values have a higher risk of fracture and thus, reduced bone quality. Some obese men and women have low BMD and not all studies showed that obesity could protect us against fracture. ${ }^{12}$ Obesity was associated with the increased levels of pancreatic hormones such as insulin, amylin and bone-anabolic preptin. ${ }^{13}$

The results of the study revealed subjects with high BMI scores have high mandibular bone density scores. These differences were influenced by several things. The sample in this study focused on men aged 5-35 years, studies focused on the elderly sample with the age range of 52-86 years, and the sample consisted of men and women.

There were 23 samples belonging to the under weight category and 7 samples belonging to over weight category. The number of samples that fell under category of underweight and overweight caused outliers data. These outliers caused abnormal research results.

\section{Conclusion}

There was no correlation between mandibular trabeculae bone density on panoramic radiograph and body mass index of men aged 5-35 years old. BMI scores increased and mandibular trabeculae bone density decreased as the age group increased.

\section{Acknowledgment}

The authors would like to thank the advisor of this paper. This study did not have financial support.

\section{Conflict of Interest}

The authors report no conflict of interest.

\section{References}

1. Avdagić SC, Barić IC, Keser I, et al. Differences in peak bone density between male and female students. Arh Hyg Rada Toxicol 2009;60: 79-86.

2. Tizabi A, Mahdavinejad R, Azizi A, et al. Correlation between height, weight, bmi with standing thoracic and lumbar curvature in growth ages. World J Sport Sci 2012;7: 54-56.

3. Gulsahi A, Paksoy CS, Ozden S, et al. Assessment of bone mineral density in the jaws and its relationship to radiomorphometric indices. Dentomaxillofac Radiol 2010;39: 284-289.

4. Smartt JM, Low Dw, Bartlett SP. The pediatric mandible: a primer on growth and development. Plast Reconstr Surg 2005;116: 14e-23e.

5. Compston JE. Sex steroids and bone. Physiol Rev 2001;81: 419-447.

6. Sochung Chung, MD P. Body mass index and body composition scaling to height in children and adolescent. Ann Pediatr Endocrinol Metab 2015;20: 125-129.

7. Rieuwpassa IE, Asmawati. Thickness cortex mandible on Toraja viewed from women's groups radiograph panoramic. J Dentomaxillofac Sci 2018;3: 41-45.

8. Camargo J, Rodriguez A, Côrtes G, et al. Analysis of bone quality on panoramic radiograph in osteoporosis research by fractal dimension. Appl Math 2016;3: 375-386.

9. Demontiero O, Vidal C, Duque G. Aging and bone loss: new insights for the clinician. Ther Adv Musculoskelet Dis 2012;4: 61-76.

10. Malina R. Normal weight gain in growing children. Heal Weight J 1999;13: 13-14.

11. Stang J, Story M. Adolescent growth and development. Guidel Adolesc Nutr Serv 2005: 1-8.

12. Iwaniec UT, Turner RT. Influence of body weight on bone mass, architecture and turnover. J Endocrinol 2016;230: R115-R130.

13. Shapses SA, Sukumar D. Bone metabolism in obesity and weight loss. Annu Rev Nutr 2014;113: 287-309.

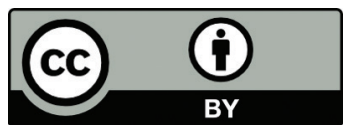

This work is licensed under a Creative Commons Attribution 